examined. Multivariable logistic regression models were used to analyse the socio-demographic, sexual behaviour and sex-work related characteristics related to the prevalence of each pathogen. Sampling weights and appropriate survey methods were utilised in regression models to account for a complex sampling design.

Results The total sample size was 2745. The average age of clients was 30.4 years (SE-0.3). Across the total sample, the prevalences of HIV, HSV-2, syphilis and CT/GC were $5.6 \%, 28.4 \%, 3.6 \%$ and $2.2 \%$, respectively. The prevalence of HIV/STIs varied substantially across districts, reaching statistical significance for HIV $(p<0.0001)$ and CT/GC ( $p=0.005)$. In multivariable models, duration of paying for commercial sex was associated with increased risk for HIV and HSV2 (both AORs-1.1; 95\% CI 1.0 to 1.1, p<0.0001). Clients using brothels as a main FSW solicitation site were associated with increased risk of HIV (AOR-2.4; 95\% CI 1.2 to 4.7, $\mathrm{p}=0.001$ ), while those frequenting lodges were at increased risk for CT/GC (AOR-6.3; 95\% CI 1.9 to 20.6, p=0.03). Clients with HSV-2 infection were at substantially higher risk of being HIV-positive (AOR-10.4; 95\% CI 6.1 to $17.7, \mathrm{p}<0.0001$ ).

Conclusions This study fills in important gaps in knowledge regarding clients of FSWs in Southern India. FSW clients clearly constitute an important bridging population between FSWs and their other sexual partners in the population. It is important to design and implement effective prevention and care programs for this well-hidden population.

\section{P1-S2.02 HIV PREVALENCE IN FEMALE SEX WORKERS IN A FOCUSED HIV PREVENTION PROJECT IN MUMBAI AND THANE DISTRICT, INDIA}

doi:10.1136/sextrans-2011-050108.59

S Bhardwaj, S Roy, S Gaikwad, V Ranebennur. Family Health International, Mumbai, India

Background Since 2004, FHI has been implementing Aastha, a Bill \& Melinda Gates Foundation supported HIV/STI prevention project for 24000 sex workers (SWs) in Mumbai and Thane. It provides STI services to approximately 11000 SWs monthly. HIV counselling and testing services were strengthened from March 2009 and by October 2010, 13669 individuals had been tested for HIV. This abstract discusses the prevalence of HIV in female sex workers (FSWs) registered with the project by age and typology. When the project commenced its intervention in 2004, the HIV prevalence in brothelbased FSWs according to the HIV Sentinel Survey 2004 was $44.76 \%$. Methods From March 2009 to October 2010, 13669 FSWs underwent voluntary counselling and testing for HIV. The results were analysed across age and typology. HIV tests were conducted and interpreted as per Indian national guidelines using three rapid tests. Results Of the 13669 tested, 623 individuals tested HIV-positive for HIV (4.6\%). Among the 623 HIV-positive individuals, 177 individuals $(28.4 \%)$ were aged $18-24$ years; 404 individuals (64.8\%) were aged 25-36 years and 42 individuals (6.7\%) were aged 37 years or older. By FSW typology, HIV prevalence was as follows-13.1\% brothel-based; $3.3 \%$ bar-based; $4.7 \%$ street-based; $1.6 \%$ home-based; and $1.6 \%$ lodge-based. The difference in HIV prevalence is statistically highly significant across different typologies $(p=0.0001)$. All the sex workers who tested HIV-positive and were willing to disclose their status were linked to care, support and treatment services.

Conclusions In this study, overall HIV prevalence among sex workers was $4.6 \%$. The highest HIV prevalence (13.1\%) was observed in the brothel-based SWs. A change in the national policy for HIV testing for brothel-based sex workers from biannually to quarterly is recommended. Historically, in Maharashtra, it has been observed that brothel-based sex workers have high incidence and this has been corroborated even after scaling up testing in this group. Given this scenario and considering the duration of the window period in any high-risk group, we can inform policy-makers to increase the frequency of HIV testing. This will provide an opportunity for early testing and inclusion of newly identified HIVpositives for referral for care and treatment.

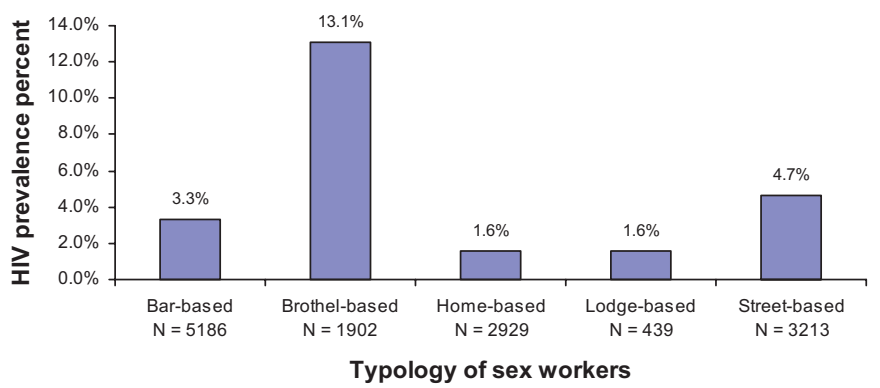

Abstract P1-S2.02 Figure 1 HIV prevalence by SW typology.

\section{P1-S2.03 DETERMINANTS OF CONDOM BREAKAGE AMONG FEMALE SEX WORKERS IN KARNATAKA, INDIA}

doi:10.1136/sextrans-2011-050108.60

${ }^{1} \mathrm{~J}$ Bradley, ${ }^{2} \mathrm{~S}$ Rajaram, ${ }^{1} \mathrm{M}$ Alary, ${ }^{2} \mathrm{~S}$ Isac, ${ }^{3} \mathrm{~S}$ Moses, ${ }^{2} \mathrm{~B} \mathrm{M}$ Ramesh. ${ }^{1}$ Laval University, Bangalore, India; ${ }^{2}$ Karnataka Health Promotion Trust, Bangalore, India; ${ }^{3}$ University of Manitoba, Winnipeg, Canada

Background Condoms are effective in preventing the transmission of HIV and other sexually transmitted infections, when properly used. However, recent data from surveys of female sex workers (FSWs) in the state of Karnataka in South India, suggest that condom breakage rates may be quite high. It is important therefore to quantify condom breakage rates, and examine what factors might precipitate condom breakage, so that programmers can identify those at risk, and develop appropriate interventions.

Methods We explored determinants of reported condom breakage in the previous month among 1928 female sex workers in four districts of Karnataka using data from cross-sectional surveys undertaken from July 2008 to February 2009. Using stepwise multivariate logistic regression, we examined the possible determinants of condom breakage controlling for several independent variables including the district and client load.

Results Overall, $11.4 \%$ of FSWs reported at least one condom break in the previous month. FSWs were much more likely to report breakage if under 20 years of age (OR 3.43, p=0.005); if divorced/ separated/widowed (OR 1.52, $\mathrm{p}=0.012$ ); if they were regular alcohol users (OR 1.63, $\mathrm{p}=0.005)$; if they mostly entertained clients in lodges/rented rooms (OR 2.99, $\mathrm{p}=0.029$ ) or brothels (OR 4.77, $\mathrm{p}=0.003)$, compared to street based sex workers; if they had ever had anal sex (OR 2.03, $\mathrm{p}=0.006)$; if the sex worker herself (as opposed to the client) applied the condom at last use (OR 1.90, $\mathrm{p}<0.001)$; if they were inconsistent condom users ( $O R$ 2.77, $p<0.001)$; and if they had never seen a condom demonstration (OR 2.37, $\mathrm{p}<0.001$ ). Conclusions The reported incidence of condom breakage was high in this study, and this is a major concern for HIV/STI prevention programs, for which condom use is a key prevention tool. Younger and more marginalised female sex workers were most vulnerable to condom breakage. Special effort is therefore required to seek out such women and to provide information and skills on correct condom use. More research is also needed on what specific situational parameters might be important in predisposing to condom breakage. 